\title{
Antigenic and Genomic Relatedness among Ehrlichia risticii, Ehrlichia sennetsu, and Ehrlichia canis $\dagger$
}

\author{
BASAVARAJU SHANKARAPPA, $\ddagger$ SUKANTA K. DUTTA, ${ }^{*}$ AND BONNIE L. MATTINGLY-NAPIER \\ Virginia-Maryland Regional College of Veterinary Medicine, University of Maryland, College Park, Maryland 20742
}

\begin{abstract}
Antigenic and genomic relatedness among Ehrlichia risticii, E. sennetsu, and $E$. canis was analyzed by enzyme-linked immunosorbent assay, Western blotting (immunoblotting) and DNA-DNA hybridization. $E$. risticii and $E$. sennets $u$ were serologically related, and their Western blot antigen profiles were nearly identical. Two antigens of $E$. sennets $u$ corresponding to the 28- and 51-kDa antigens of $E$. risticii were apparently larger than the $E$. risticii antigens, and the 55-kDa antigen of $E$. risticii appeared to be unique to this species. The 110-, 70-; and 44-kDa antigens of these two species were identical, as determined by the use of monospecific antibodies. DNA homology between these two species was high. On the other hand, $E$. canis was antigenically least reactive with the antisera to $E$. risticii and $E$. sennetsu. However, a dog convalescent-stage $E$. canis antiserum recognized antigens in the other two species which were different from those recognized by their homologous antisera. Similarly, homology between the DNA of $E$. canis and the DNAs of the other two species was very minimal. These results indicate that $E$. risticii and $E$. sennetsu are closely related both at the genomic and antigenic levels and that the relationship of these two species with $E$. canis is minimal.
\end{abstract}

Members of the family Ehrlichieae belong to a group of obligate intracellular parasites with complex mechanisms of growth and multiplication which are poorly understood. Ehrlichia risticii was identified in 1985 as the causative agent of Potomac horse fever $(3,8,16)$. It has recently been reported that antibodies to $E$. risticii are common in the United States and Europe (7a, 18). E. sennetsu is the causative agent of human sennetsu fever, found mainly in western Japan, while $E$. canis causes canine ehrlichiosis (tropical canine pancytopenia) and occurs worldwide (17, 20). One common feature among these ehrlichieae is their predilection for the monocytic series of cells in their respective hosts (17).

The renewed interest in the study of Ehrlichieae is due to the recent identification of $E$. risticii, serologic evidence of an association of human ehrlichiosis with $E$. canis $(7,11,14)$, and recent isolation of the etiological agent from humans with ehrlichiosis (1a). The ability of $E$. risticii and $E$. sennetsu to experimentally infect nonhuman primates (22a) and the economic considerations of Potomac horse fever and canine ehrlichiosis are the other reasons for this interest.

Present knowledge of the relatedness among Ehrlichieae is based on similarity in morphologic and biologic characteristics and cross-reactivities of antisera $(8,15,19)$. The use of modern approaches in the identification of cross-reacting antigens and quantitation of serum cross-reactivity allows a reevaluation of the relatedness among these species in greater detail. Because the analysis of phenotypic expression involves less than $20 \%$ of the total genomes of the bacteria (1), it is necessary and now possible to evaluate these relationships at the genomic level. Toward these goals, we report the reactivities of species-specific sera by enzymelinked immunosorbent assay (ELISA), comparative analyses of the protein-antigen composition, and the relatedness of DNA among the three species of Ehrlichia.

\footnotetext{
* Corresponding author.

$\dagger$ Published as scientific article no. A6252, contribution no. 8421 of the Maryland Agricultural Experiment Station.

$\ddagger$ Present address: Department of Pathology, School of Medicine University of Pittsburgh, Pittsburgh, PA 15261.
}

\section{MATERIALS AND METHODS}

Ehrlichial organisms and purification. E. risticii, isolated in our laboratory (3), and E. sennetsu (Miyayama strain; provided by G. Dasch, Naval Medical Research Institute, Bethesda, Md.) were propagated in human histiocyte (ATCC $\mathrm{U}-937$ ) and mouse macrophage (ATCC P-388D $\mathrm{D}_{1}$ ) cells, respectively, in RPMI 1640 medium supplemented with $10 \%$ fetal calf serum and $4 \mathrm{mM}$ L-glutamine. E. canis (Florida strain; provided by C. Holland, University of Illinois, Urbana) was grown in primary canine monocytic cells in medium 199 supplemented with $20 \%$ dog serum (12). The in vitro infection process was monitored by acridine orange (10) and Giemsa staining.

The method of purifying organisms over a 20 to $45 \%$ Renografin linear density gradient $(25,26)$, as previously described for $E$. risticii (4), was adhered to with slight modification. This modification included digestion of the preparations with DNAse I (final concentration, $1 \mu \mathrm{g} / \mathrm{ml}$; Bethesda Research Laboratories, Gaithersburg, Md.) for 10 min prior to loading it on the gradient. This procedure eliminated host cell DNA strands in the gradient, which were otherwise present. Both $E$. sennetsu and $E$. canis yielded a Renografin banding pattern similar to that of $E$. risticii. However, for E. canis, because of limitations of the total yield of infected primary canine monocyte cultures and the lack of previous knowledge of the banding pattern, fractions collected from the gradient were analyzed by conducting an indirect ELISA (22) using dog anti-E. canis serum (provided by $C$. Holland). The peaks of immune reactivity corresponded to the banding patterns observed for other ehrlichieae, and these peak fractions were pooled and processed as described previously (4). The protein concentration of each of the purified organism preparations was estimated by a bicinchoninic acid protein assay system (Pierce Chemical Co., Rockford, Ill.) with bovine serum albumin as the standard. For DNA studies, the organisms were suspended in TNE buffer (10 mM Tris [pH 8], $150 \mathrm{mM} \mathrm{NaCl}, 1 \mathrm{mM}$ EDTA).

Antisera. Production of rabbit anti-E. risticii serum has been described previously (6). Antisera to E. sennetsu and $E$. canis were produced by injecting rabbits intramuscularly 
with purified organisms by the following slight modification to this protocol. The first injection contained $400 \mu \mathrm{g}$ of the antigen mixed in Freund's complete adjuvant. The second injection, administered 2 weeks later, contained $200 \mu \mathrm{g}$ of the antigen mixed in Freund's incomplete adjuvant, and the third injection, given at 4 weeks, contained $200 \mu \mathrm{g}$ of the antigen alone. Test bleedings were done to evaluate the antibody reactivities by ELISA and Western blot (immunoblot), and final bleedings were done at 6 weeks postimmunization. A Western blot analysis for $E$. canis was first done with the pre- and postinfection dog antisera provided by $C$. Holland to accurately determine the antigen profile of $E$. canis. A convalescent-stage dog antiserum to $E$. canis (provided by E. H. Stephenson, University of Maryland, College Park), used in all further analyses, was validated by ascertaining the Western blot reactivity that was identical to that observed with the postinfection antiserum provided by Holland.

Affinity-purified recombinant antigen-selected monospecific antibodies to the 110-, 70-, and 44-kDa antigens of $E$. risticii were prepared by using rabbit anti-E. risticii serum and the lambda gt11 recombinant clones expressing these antigens $(5,21 \mathrm{a})$. Monospecific antiserum to the $55-\mathrm{kDa}$ antigen was obtained from mice immunized with the purified recombinant antigen (5; unpublished data).

Extraction of DNA. Chromosomal-DNA extraction from the purified organisms was done as described previously (6) with few modifications. Prior to DNA extraction, $\mathrm{MgSO}_{4}$ to $10 \mathrm{mM}$ and DNAse I (BRL) to $1 \mu \mathrm{g} / \mathrm{ml}$ were added, and the mixture was incubated at $37^{\circ} \mathrm{C}$ for $30 \mathrm{~min}$. Next, the DNAse was inhibited by the addition of EDTA to $20 \mathrm{mM}$, and the DNA was extracted by using sodium dodecyl sulfate (SDS), proteinase $\mathrm{K}$, phenol-chloroform extraction, and RNase $\mathrm{T}_{1}$ treatments. The DNA was quantitated with a TKO 100 DNA fluorometer (Hoeffer Scientific Instruments, San Francisco, Calif.).

PAGE and Western blotting. Polyacrylamide gel electrophoresis (PAGE) and Western blotting have been described previously (22). For use in Western blotting, identical amounts of the total protein from each species were loaded, and each of the antisera was diluted 1:50 in casein solution. Uninfected human histiocyte, mouse macrophage, and canine monocyte cells were used for Western blots with each of the antisera to determine reactivity with host cell components. Monospecific antibodies were evaluated for their reactivities with the three ehrlichial species in the Western blot assay as previously described (5).

ELISA. The cross-reactivities of native antigens were evaluated by ELISA using purified ehrlichial preparation as the antigen by the procedure described previously (22). Titers of each serum with respect to homologous and heterologous antigens were determined. Optimal binding with the respective homologous systems observed at a serum dilution of 1:100 was used to evaluate the homologous and heterologous sensitivities at lower antigen concentrations.

DNA-DNA hybridizations. Blotting and hybridization experiments were conducted on GeneScreen Plus membranes according to the procedures suggested by the manufacturer (NEN Products, Boston, Mass.). The chromosomal DNA was denatured in $0.25 \mathrm{~N} \mathrm{NaOH}$ and diluted in $0.125 \mathrm{~N}$ $\mathrm{NaOH}-0.125 \times \mathrm{SSC}(1 \times \mathrm{SSC}$ is $0.15 \mathrm{M} \mathrm{NaCl}$ and $0.015 \mathrm{M}$ sodium citrate) to obtain twofold dilutions starting with 100 ng. Salmon sperm DNA (Sigma) and DNA from U-937 and $\mathbf{P}-\mathbf{3 8 8 D}_{1}$ cells were used as controls. Radiolabeling of DNA was performed by nick translation using a commercial kit (Bethesda Research Laboratories) and $\left[\alpha-{ }^{32} \mathrm{P}\right] \mathrm{dCTP}$ (Amer-

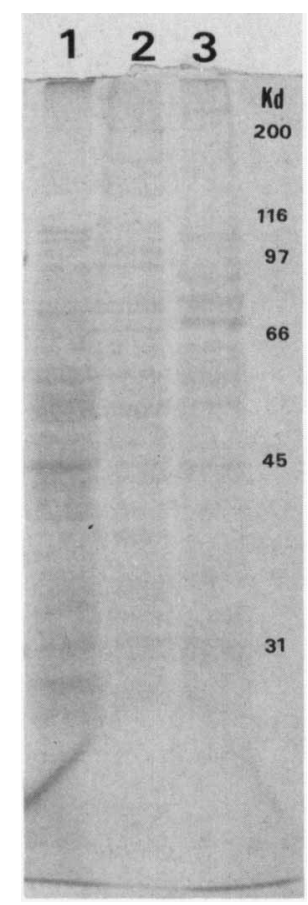

FIG. 1. PAGE of E. risticii (lane 1), E. sennetsu (lane 2), and $E$. canis (lane 3) stained with Coomassie blue. Numbers on the right indicate the position of molecular size markers in kilodaltons.

sham, Arlington Heights, Ill.). A specific activity of $10^{8}$ $\mathrm{cpm} / \mu \mathrm{g}$ of DNA was obtained for $E$. risticii, and a specific activity of $3 \times 10^{7} \mathrm{cpm} / \mu \mathrm{g}$ of DNA was obtained for the other two species because of a lower input of DNA. Prehybridization and hybridization solutions contained $5 \times$ SSC, $5 \%$ dextran sulfate, $1 \times$ Denhardt's solution, $1 \%$ SDS, and $100 \mu \mathrm{g}$ of nonhomologous DNA per ml. Following hybridizations at $65^{\circ} \mathrm{C}$ for $36 \mathrm{~h}$, the membranes were washed with $2 \times, 0.5 \times$, and $0.1 \times$ SSC containing $0.1 \%$ SDS for $30 \mathrm{~min}$ each. The wet membranes were exposed to X-Omat autoradiograph film (Kodak, Rochester, N.Y.) with intensifying screens for various time intervals.

\section{RESULTS}

Ehrlichia purification. Renografin gradient centrifugation for the purification of $E$. risticii (4), based on the technique for the purification of rickettsiae $(25,26)$, was found to be efficient for the purification of $E$. sennetsu and $E$. canis. Because of the difficulty in visualizing the bands with $E$. canis, the fractions were tested for the presence of antigen by ELISA with anti-E. canis dog serum and pooled.

Protein and antigen profiles. The electrophoretic patterns of ehrlichial proteins appeared to be distinctive for each species (Fig. 1). The Coomassie blue-stained components were relatively more similar in $E$. risticii and $E$. sennetsu than in either of these species and $E$. canis. Western blotting analyses with homologous and heterologous antisera identified the profile of the antigenic components of each organism (Fig. 2). Sixteen antigens ranging in size from 24 to $120 \mathrm{kDa}$ were identified in $E$. risticii with the homologous antisera, while nine such antigens were identified in E. sennetsu. $E$. canis antisera identified nine antigens in $E$. canis ranging in size from 30 to $138 \mathrm{kDa}$.

The Western blot reactivities of all antisera were tested 


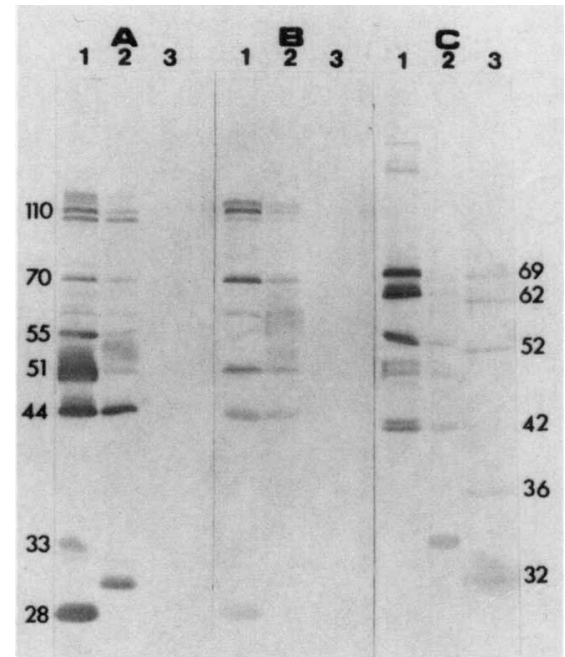

FIG. 2. Western blot analysis of E. risticii (lanes 1), E. sennetsu (lanes 2), and $E$. canis (lanes 3) with rabbit $E$. risticii antisera (A), rabbit E. sennetsu antisera (B), and dog E. canis antisera (C). Numbers indicate molecular sizes in kilodaltons.

with the host cells as internal controls (results not shown). The $E$. risticii antisera reacted with one U-937 cell protein that was slightly larger than the $70-\mathrm{kDa}$ antigen, three $\mathrm{P}-388 \mathrm{D}_{1}$ cell proteins, and none of the canine monocyte proteins. Similarly, E. sennetsu antisera reacted with several of the P-388D 1 cell proteins but none of the other host cells. The dog anti-E. canis serum reacted extensively with $\mathrm{P}-388 \mathrm{D}_{1}$ and a few U-937 cell proteins. However, none of the antigenic protein bands mentioned above were comparable to those for antigens identified in the Western blots of purified-organism preparations. Further, these antigens identified in the host cells were apparently diluted out in the purification process and hence were not identifiable in Western blots using the purified organisms.

The antigens identified in E. risticii (6) and E. sennetsu by using $E$. risticii antiserum were almost identical except for differences in the 51- to 55- and 28- to 33-kDa regions (Fig. 2A). A similar antigen profile was observed in the reactivity of $E$. sennetsu antiserum except for the difference in relative mobilities of antigens in the 55- to $70-\mathrm{kDa}$ region (panel B). Antisera to both species identified similar differences between $E$. risticii and $E$. sennetsu in the antigen profiles at the 28 - to $33-\mathrm{kDa}$ region, and neither antiserum was reactive with $E$. canis components.

E. canis exhibited a distinctive antigenic profile (Fig. 2C). The antigenic components identified by using dog anti- $E$. canis serum were fewer for $E$. canis than for the other two species with their respective antisera. This dog antiserum also reacted quite extensively with both $E$. risticii and $E$. sennetsu. But the molecular weights of the antigens identified in these two species were different from those observed in $E$. canis and were not comparable to those observed with the respective homologous antisera (Fig. 3A and B). The rabbit antisera produced against purified $E$. canis reacted with $E$. risticii proteins migrating at 51 and $28 \mathrm{kDa}$, while no such reactivity was seen with $E$. sennetsu (results not shown). However, a majority of the antigens identified in $E$. canis with this serum were analogous to those observed in the host canine monocytic cell components (results not shown), and this serum was therefore not used in further

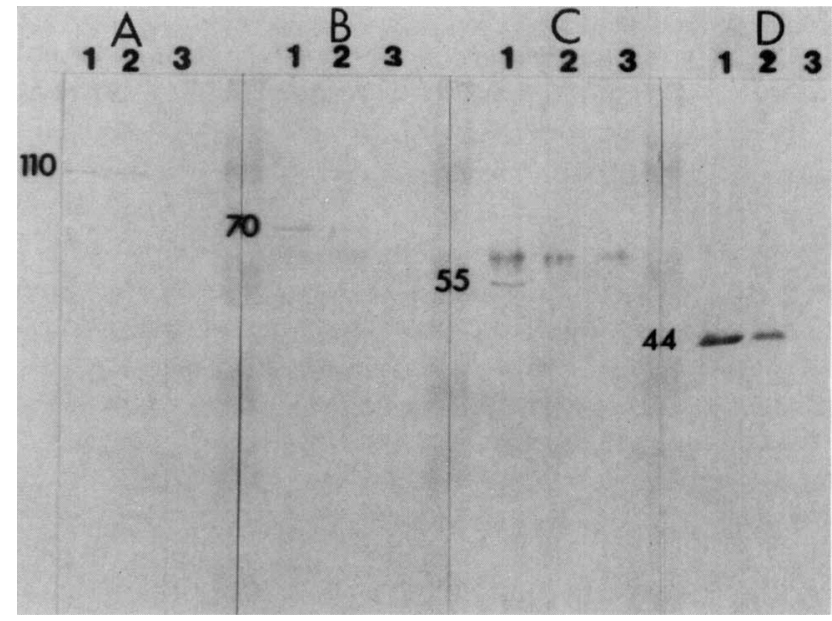

FIG. 3. Western blot analyses of E. risticii (lanes 1), E. sennetsu (lanes 2), and E. canis (lanes 3) using monospecific antibodies to recombinant antigens of $E$. risticii (numbers correspond to the molecular sizes of the antigens in kilodaltons). Antibody to the $55-\mathrm{kDa}$ antigen was obtained from mice immunized with this recombinant antigen. All the other antibodies were affinity purified by using the rabbit $E$. risticii antisera and the recombinantly expressed antigen. A nonspecific band was observed in the region between the 55- and 70-kDa antigens in many blots

analyses. Normal dog and rabbit sera were not reactive with any ehrlichial species.

The reactivities of monospecific antibodies to the 110-, 70-, and 44-kDa recombinant antigens of $E$. risticii indicated the presence of identically sized proteins in E. sennetsu (Fig. 3 ). However, monospecific antisera to the $55-\mathrm{kDa}$ antigen of $E$. risticii failed to react with any antigen of $E$. sennetsu, and none of these monospecific antibodies reacted with $E$. canis.

ELISA reactivity. The ELISA results with homologous and heterologous systems are presented in Table 1. Both anti-E. risticii and anti-E. sennetsu sera exhibited high homologous titers along with an extensive cross-reactivity between them. The magnitude of homologous ELISA titers of these sera indicated the presence of species-specific determinants, while the extent of cross-reactivity was an indication of the presence of a large pool of shared determinants. Antisera to both of these organisms had minimal cross-reactivity with $E$. canis, with $E$. sennetsu antisera exhibiting a slightly higher titer. Dog anti-E. canis serum reacted moderately with $E$. risticii and to a fourfold-lesser titer with $E$. sennetsu. Rabbit anti-E. canis serum exhibited a slight reactivity with both $E$. risticii and $E$. sennetsu.

TABLE 1. Serologic relatedness among E. risticii, E. sennetsu, and E. canis

\begin{tabular}{lrrr}
\hline \multirow{2}{*}{ Antiserum } & \multicolumn{3}{c}{ Titer for antigen from $^{a}$ : } \\
\cline { 2 - 4 } & E. risticii & E. sennetsu & E. canis \\
\hline Rabbit anti-E. risticii & 102,400 & 12,800 & $<50$ \\
Rabbit anti-E. sennetsu & 25,600 & 102,400 & 200 \\
Rabbit anti-E. canis & 800 & 800 & 51,200 \\
Dog anti-E. canis & 6,400 & 1,600 & $51,200^{b}$ \\
\hline
\end{tabular}

a Numbers represent the reciprocal dilution of the serum when the reactivity was equivalent to the background optical density.

${ }^{b}$ A higher background was observed because of the use of canine monocyte-derived antigen and anti-dog immunoglobulin second antibody. 


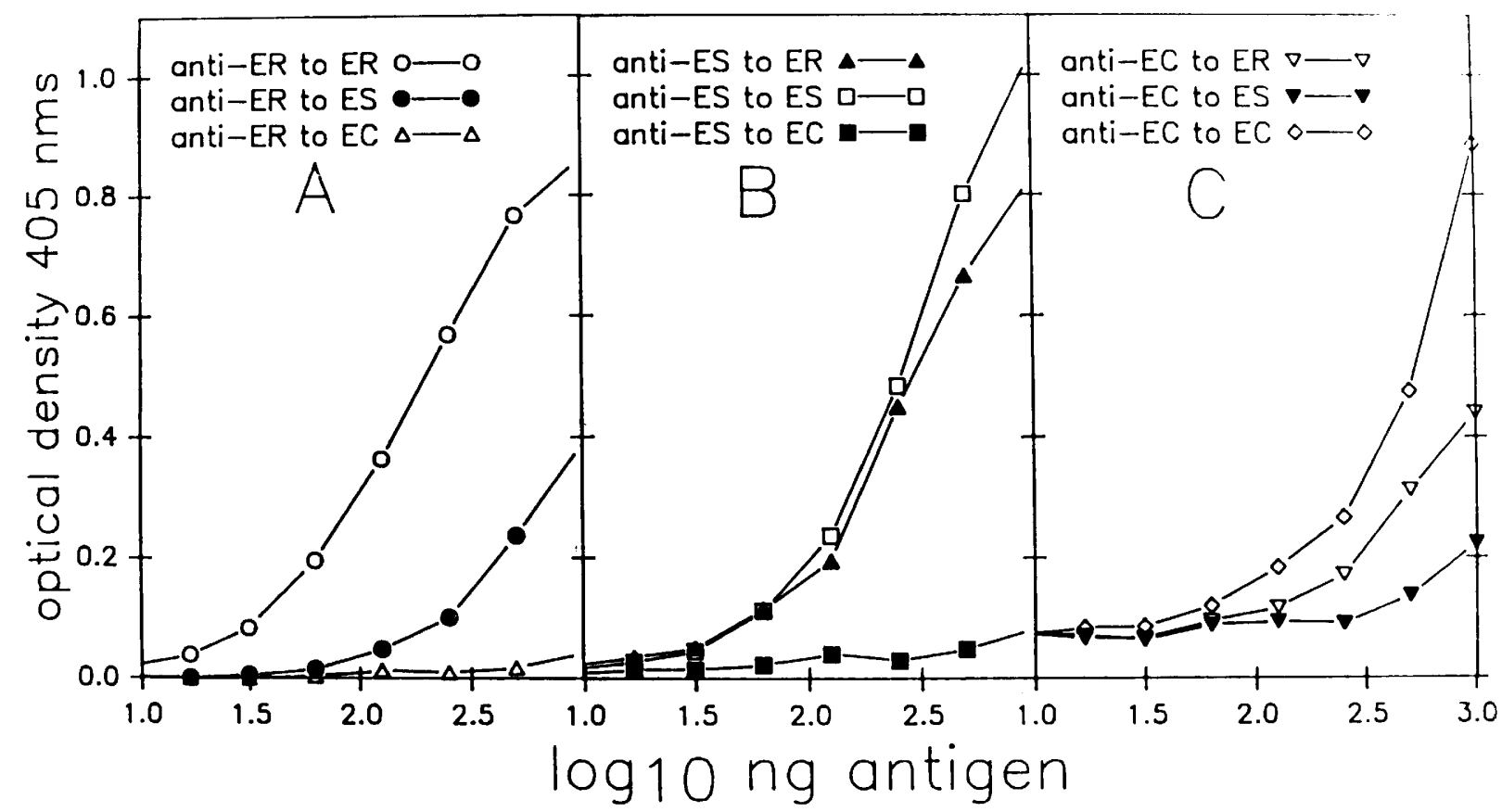

FIG. 4. Comparison of ELISA reactivities of rabbit E. risticii (ER) (A), rabbit E. sennetsu (ES) (B), and dog E. canis (EC) (C) antisera with limiting dilutions of each organism. The organisms were serially diluted and reacted with a 1:100 dilution of each antiserum.

When the ehrlichial antigens were serially diluted to determine the lower limits at which each serum could detect the homologous and heterologous antigens, the ELISA patterns were similar (Fig. 4) to those observed in the ELISA described above (Table 1). Distinguishing between $E$. risticii and $E$. sennetsu was easier with antisera to $E$. risticii. Both of these sera reacted minimally with $E$. canis. The dog anti-E. canis serum exhibited a hierarchy in its ability to detect a smaller amount of $E$. risticii than of $E$. sennetsu.

DNA relatedness. DNA-DNA dot hybridization studies (Fig. 5) demonstrated extensive homology between $E$. risticii and $E$. sennetsu. At concentrations of DNA used in these blots, hybridization signals obtained from the labeled $E$. risticii DNA with $E$. canis DNA were only faintly visible in the autoradiogram (Fig. 5A), while no similar signal was observed with labeled $E$. sennetsu DNA. Because of the low specific activity of the $E$. canis DNA probe, the reciprocal hybridization signals could not be identified.

\section{DISCUSSION}

While comparative structural studies on the rickettsial group of organisms have been reported $(13,24)$, such studies with ehrlichial organisms are lacking. Most of the studies on $E$. canis, which is the type species (20), were stimulated by the severe epizootic of canine ehrlichiosis in military dogs in Vietnam from 1968 to 1970 (17). The recent emergence of Potomac horse fever and the isolation of the etiological agent of human ehrlichiosis signify the need to study these species in greater detail.

Our demonstration of cross-reactivity between $E$. risticii and $E$. sennetsu supports the reported serologic relationship between these two species $(9,15,19)$. Also, we have identified the antigenic constituents apparently responsible for this cross-reactivity and demonstrated equivalent DNA homologies. The antigen profile of $E$. sennetsu is in general agreement with one report identifying 100-, 80-, 52-, and 46-kDa antigens of $E$. sennets $u$ with patient sera (23). While similar antigenic profiles were observed with $E$. risticii and $E$. sennetsu, the differences in this antigenic profile in the 51to $70-$ and 28- to $33-\mathrm{kDa}$ regions (Fig. $3 \mathrm{~A}$ and $\mathrm{B}$ ) suggest an important distinction. From the pattern of reactivity of $E$. risticii antiserum with the 51-, 49-, and $28-\mathrm{kDa} E$. risticii antigens and their apparent counterparts in E. sennetsu, it appears that analogous proteins are present in E. sennetsu but are slightly larger. Furthermore, this pattern has been identical in the 28- to $33-\mathrm{kDa}$ region with both antisera. However, a monoclonal antibody to the $28-\mathrm{kDa} E$. risticii antigen was not reactive with $E$. sennetsu (22). The use of monospecific antibodies has demonstrated the analogous reactivities and sizes of the 110-, 70-, and 44-kDa antigens in $E$. risticii and $E$. sennetsu, while such a relationship was not observed for the $55-\mathrm{kDa}$ antigen.

It is possible that some of the differences identified in the reactivities of antisera can be attributed to qualitative differences in purified ehrlichial preparations. Because of differences in the growth of $E$. risticii in suspension culture compared with that of monolayer and primary cell cultures of other ehrlichieae, it is possible to obtain a highly enriched preparations of $E$. risticii compared with preparations of the other species. However, we believe that such differences are only minor and do not contradict any of the observed results.

Species-specific antibodies to E. risticii and E. sennetsu failed to substantiate the relationship between these species and $E$. canis to the extent implied in previous studies (19, 21). While the normal dog sera did not react with any ehrlichieae, the $\operatorname{dog} E$. canis antiserum has shown an interesting reactivity with other species. Because this crossreactivity is with antigens that were not identified by the homologous antisera and is not observed with normal dog and rabbit $E$. canis hyperimmune sera, we believe this cross-reactivity is of nonspecific origin. In a previous study, 


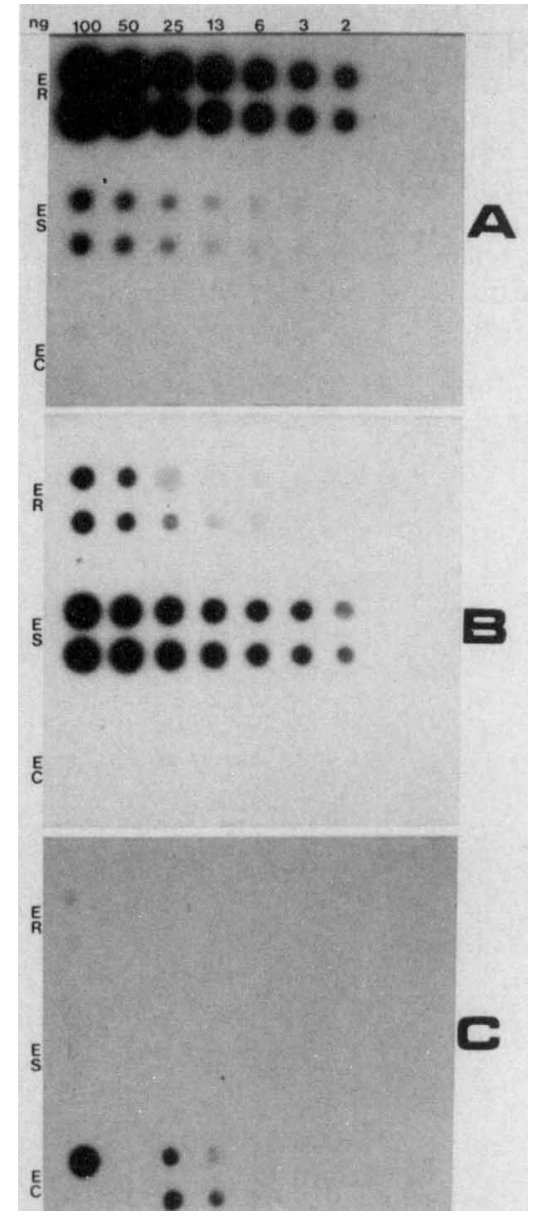

FIG. 5. DNA-DNA dot blot hybridization analysis of E. ristici (ER), E. sennetsu (ES), and E. canis (EC) DNAs. Numbers at the top indicate the total amount of DNA (in nanograms) applied to the membrane. Radiolabeled E. risticii, E. sennetsu, and E. canis DNAs were used as probes in panels A, B, and C, respectively. E. canis DNA was added to the one 100-ng well, was absent in the 50-ng wells, was added to all other wells in panel $C$, and was added to all wells in panels A and B.

we had identified antibodies in the preinfective sera of horses that were reactive with SDS-denatured antigens in Western blots of $E$. risticii (2). Hence, we believe more studies are needed to assess the presence of normal antibodies reactive with various ehrlichial species prior to unambiguous interpretation of cross-reactivity.

The relationship between $E$. risticii and $E$. sennetsu observed in their DNAs substantiates the extent of relationship observed by using the antibodies. Similarly, a lack of extensive DNA homology of these species with $E$. canis is analogous to the antigenic relationship observed with the antisera to purified organisms. In conclusion, it appears that $E$. risticii and $E$. sennetsu are highly related at both the genomic and the antigenic levels, while $E$. canis seems to be minimally related to these species.

\section{REFERENCES}

1. Brenner, D. J. 1983. Impact of modern taxonomy on clinical microbiology. ASM News 49:58-63.

1a.Dawson, J., B. Anderson, D. Fishbein, J. Sanchez, C. Goldsmith, K. Wilson, and C. Duntley. 1991. 9th Sesquiannu. Meet. Am. Soc. Rickettsiol. Rickettsial Dis., abstr. 11, p. 20.
2. Dutta, S. K., B. L. Mattingly, and B. Shankarappa. 1989. Antibody response to Ehrlichia risticii and antibody reactivity to component antigens in horses with induced Potomac horse fever. Infect. Immun. 57:2959-2962.

3. Dutta, S. K., A. C. Myrup, R. M. Rice, M. G. Robl, and R. C. Hammond. 1985. Experimental reproduction of Potomac horse fever in horses with newly isolated ehrlichia organism. J. Clin. Microbiol. 22:265-269.

4. Dutta, S. K., R. M. Rice, T. D. Hughes, P. K. Savage, and A. C. Myrup. 1987. Detection of serum antibodies against Ehrlichia risticii in Potomac horse fever by enzyme-linked immunosorbent assay. Vet. Immunol. Immunopathol. 14:85-92.

5. Dutta, S. K., B. Shankarappa, and B. L. Mattingly-Napier. 1991. Molecular cloning and analysis of recombinant major antigens of Ehrlichia risticii. Infect. Immun. 59:1162-1169.

6. Dutta, S. K., B. Shankarappa, S. R. Thaker, and B. L. Mattingly-Napier. 1990. DNA restriction endonuclease cleavage pattern and protein antigen profile of Ehrlichia risticii. Vet. Microbiol. 25:29-38.

7. Harkess, J. R., S. A. Ewing, J. M. Crutcher, J. Kudlac, G. McKee, and G. R. Istre. 1989. Human ehrlichiosis in Oklahoma J. Infect. Dis. 159:576-579.

7a.Holland, C. J. 1988. Annu. Symp. Potomac Horse Fever, Laurel, Md., 24 May 1988

8. Holland, C. J., M. Ristic, A. I. Cole, P. Johnson, G. Baker, and T. Goetz. 1985. Isolation, experimental transmission and characterization of the causative agent of Potomac horse fever Science 277:522-526.

9. Holland, C. J., E. Weiss, W. Burgdorfer, A. I. Cole, and I. Kakoma. 1985. Ehrlichia risticii sp. nov.: etiological agent of equine monocytic ehrlichiosis (synonym, Potomac horse fever) Int. J. Syst. Bacteriol. 35:524-526.

10. Lauer, B. A., L. B. Reller, and S. Mirrett. 1981. Comparison of acridine orange and gram stains for detection of microorganisms in cerebrospinal fluid and other clinical specimens. J. Clin. Microbiol. 14:201-215.

11. Maeda, K., N. Markowitz, R. C. Hawley, M. Ristic, D. Cox, and J. E. McDade. 1987. Human infection with Ehrlichia canis, a leukocytic rickettsia. N. Engl. J. Med. 316:853-856.

12. Nyindo, M. B. A., M. Ristic, D. L. Huxsoll, and A. R. Smith. 1971. Tropical canine pancytopenia-in vitro cultivation of the causative agent, Ehrlichia canis. Am. J. Vet. Res. 32:16511658.

13. Oaks, E. V., R. M. Rice, D. J. Kelly, and C. K. Stover. 1989. Antigenic and genetic relatedness of eight Rickettsia tsutsugamushi antigens. Infect. Immun. 57:3116-3122.

14. Peterson, L. R., L. A. Sawyer, D. B. Fishbein, P. W. Kelley, R. J. Thomas, L. A. Magnarelli, M. Redus, and J. E. Dawson. 1989. An outbreak of ehrlichiosis in members of an army reserve unit exposed to ticks. J. Infect. Dis. 159:562-568.

15. Rikihisa, Y. 1991. The tribe Ehrlichieae and ehrlichial diseases. Clin. Microbiol. Rev. 4:286-308.

16. Rikihisa, Y., and B. D. Perry. 1985. Causative ehrlichial organisms in Potomac horse fever. Infect. Immun. 49:513-517.

17. Ristic, M. 1986. Pertinent characteristics of leukocytic rickettsiae of humans and animals, p. 182-187. In L. Leive (ed.) Microbiology-1986. American Society for Microbiology, Washington, D.C

18. Ristic, M. 1990. Current strategies in research on ehrlichiosis, $p$ 136-153. In J. C. Williams and I. Kakoma (ed.), Ehrlichiosis: a vector-borne disease of animals and humans. Kluwer Academic Publishers, Boston.

19. Ristic, M., C. J. Holland, J. E. Dawson, J. Sessions, and J. Palmer. 1986. Diagnosis of equine monocytic ehrlichiosis (Potomac horse fever) by indirect immunofluorescence. J. Am. Vet. Med. Assoc. 189:39-46.

20. Ristic, M., and D. L. Huxsoll. 1984. Ehrlichieae, p. 704-709. In N. R. Krieg and J. G. Holt (ed.), Bergey's manual of systematic bacteriology, vol. 1. The Williams \& Wilkins Co., Baltimore.

21. Ristic, M., D. L. Huxsoll, N. Tachibana, and G. Rapmund. 1981 Evidence of a serologic relationship between Ehrlichia canis and Ehrlichia sennetsu. Am. J. Trop. Med. Hyg. 30:1324-1328.

21a.Shankarappa, B. 1990. Ph.D. dissertation. University of Mary- 
land, College Park.

22. Shankarappa, B., and S. K. Dutta. 1989. Production and characterization of monoclonal antibodies to Ehrlichia risticii. Am J. Vet. Res. 50:1145-1149.

22a.Stephenson, E. H. 1990. Experimental ehrlichiosis in nonhuman primates, p. 93-99. In J. C. Williams and I. Kakoma (ed.), Ehrlichiosis: a vector-borne disease of animals and humans. Kluwer Academic Publishers, Boston.

23. Tachibana, N. 1986. Sennetsu fever: the disease, diagnosis, and treatment, p. 205-208. In L. Leive (ed.), Microbiology-1986. American Society for Microbiology, Washington, D.C.
24. Weiss, E. 1982. The biology of Rickettsiae. Annu. Rev. Microbiol. 36:345-370.

25. Weiss, E., J. C. Coolbaugh, and J. C. Williams. 1975, Separation of viable Rickettsia typhi from yolk sac and L cell host components by Renografin density gradient centrifugation. Appl. Microbiol. 30:456-463.

26. Weiss, E., G. A. Dasch, Y. Kang, and H. N. Westfall. 1988 Substrate utilization by Ehrlichia sennetsu and Ehrlichia risticii separated from host constituents by Renografin gradient centrifugation. J. Bacteriol. 170:5012-5017. 